\title{
Virtual Synthetic Aperture Radar Target Based on a Miniaturized Monostatic Digital Delay Transponder
}

\author{
Michael Stelzig, Andreas Benedikter, Ralf Horn, Marc Jäger, Martin Keller, Rolf Scheiber, \\ Gerhard Krieger, Fellow, IEEE, and Martin Vossiek, Fellow, IEEE
}

\begin{abstract}
The ice penetrating capability of low-frequency radar systems may offer a unique potential for the detection, localization, and guidance of exploratory ice penetrating probes for cryospheric applications. As these probes exhibit a comparably small radar cross section, a reliable detection, especially in high clutter surroundings, such as ice sheets, is prevented. As a solution a miniaturized monostatic digital delay transponder is developed and tested regarding its functionality with an airborne SAR sensor.
\end{abstract}

Index Terms-SAR, Radar, Transponder, Pulse Compression, Radar Cross Section.

\section{INTRODUCTION}

$\mathbf{E}$ NCELADUS Explorer (EnEx) is an initative by the German Space Agency at the German Aerospace Center (DLR) that is proposing a mission concept for the exploration of Saturn's ice covered moon Enceladus [1]. The concept includes the probing of a subglacial water reservoir using melting probes as described in [2], [3]. For safe guidance of such a probe it is mandatory to set up a reliable navigation assistance. An L-band synthetic aperture radar (SAR) system can be utilized for this purpose, offering both significant ice penetration capability and potentially high bandwidth and is therefore often used for cryospheric studies, such as in [4]-[7].

A reliable detection of small melting probes as described in [3] $(\varnothing=8 \mathrm{~cm})$ within the surrounding backscatter of the ice is not feasible as the radar cross section (RCS) is inherently small for L-band frequencies. This drives the need for an active radar transponder inside the melting probe. However, radar transponders are typically realized based on a bistatic architecture [8]-[11] which is not feasible in the limited space within a melting hole. In this work, we present the concept of a miniaturized monostatic digital delay SAR transponder and report first results from a test campaign conducted in June 2021 using DLR's airborne SAR sensor F-SAR [7], [12] which serves as a functionality validation for a further campaign planned for March 2022 on an Alpine glacier.

\section{ACTIVE RADAR TARGET}

\section{A. Requirements}

The requirements for an active radar target for the described application can be narrowed down to three main aspects:

1) size: the transponder must fit into the hole with dimensions dictated by the melting probe;

Manuscript received August 31, 2021; revised December 2, 2021. Accepted December 6, 2021. Date of publication March Any Date, 2022; December 6, 2021.
2) signal strength: the response signal must be strong enough to be reliably detected by the radar system. A signal gain $>40 \mathrm{~dB}$ is desired;

3) signal integrity: the radar pulse and its phase must be retained in order to apply SAR algorithms.

\section{B. Realization}

Monostatic architectures suffer from an inherently small isolation between transmit $(\mathrm{Tx})$ and receive $(\mathrm{Rx})$ path which is mainly dominated by the antenna mismatch and the isolation of the circulator [13]. For the presented transponder a planar Vivaldi antenna on a low cost FR4-substrate with a wide field of view and relatively low gain of approximately $G_{\text {ant }}=1.5 \mathrm{~dB}$ is used. As shown in Fig. 1, the antenna has an even wider bandwidth than required by the used radar with the parameters as shown in Table I. This prevents a large antenna mismatch due to the ice of potentially unknown permittivity in the near field of the antenna. Thus, to achieve a high RCS $\sigma_{\operatorname{Tr}}$ it is mandatory to realize a significant system gain $G_{\mathrm{sys}}$ as can be derived from [14]

$$
\sigma_{\mathrm{Tr}}=G_{\mathrm{ant}}^{2} \cdot G_{\mathrm{sys}} \cdot \frac{\lambda^{2}}{4 \pi} .
$$

To realize a $G_{\text {sys }}$ beyond $40 \mathrm{~dB}$, Tx and Rx paths must be further isolated to prevent oscillations or saturation. This is achieved by integrating a digital delay between the input

TABLE I: Configuration of the F-SAR L-band radar

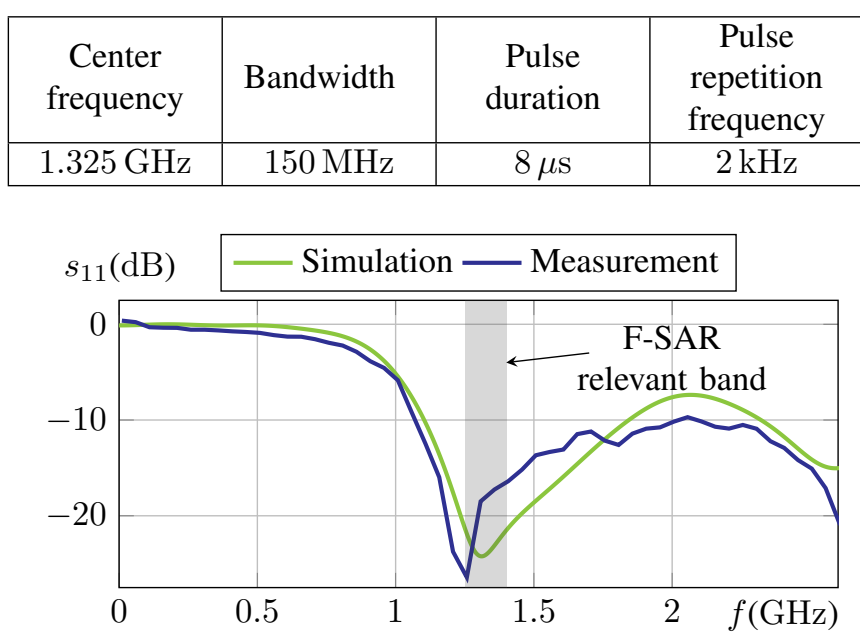

Fig. 1: Comparison between simulated and measured $s_{11}$ of the realized Vivaldi antenna. 


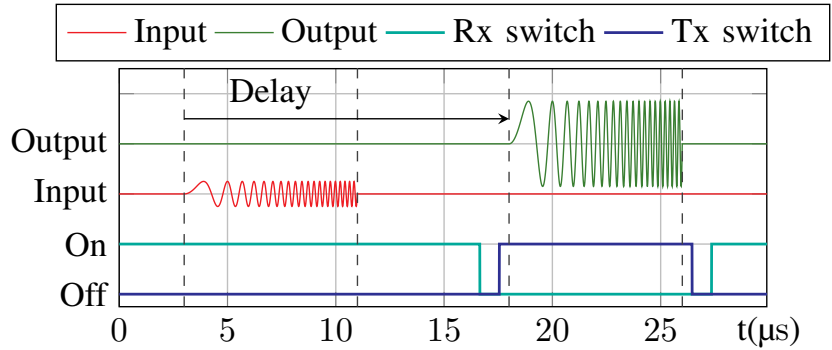

Fig. 2: Input and time shifted, amplified output signal of the transponder as well as timed Rx/Tx switching.

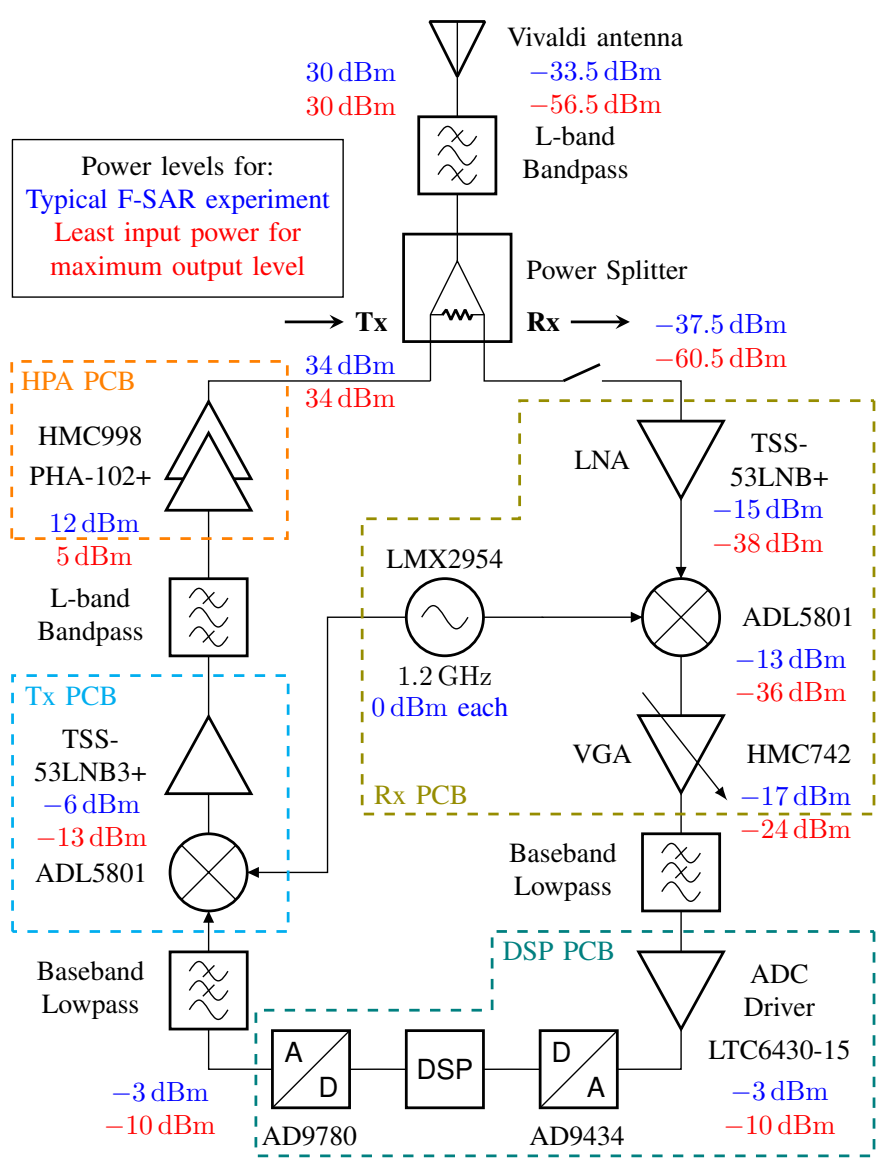

Fig. 3: System structure and power levels of the developed radar transponder.

and output of the transponder while $\mathrm{Rx}$ and $\mathrm{Tx}$ paths are switched on and off accordingly. This is visualized in Fig. 2 and can be realized with a system structure as shown in Fig. 3 . A first prototype of the transponder hardware is shown in Fig. 4. Fig. 3 additionally shows the power levels throughout the transponder for a typical F-SAR experiment in blue. As moisture level and density of the ice is not known in advance, the additional attenuation and hence, the received power for the in-ice use case cannot be predicted. Therefore, the minimum input power level required for achieving the maximum output power is also displayed in red in Fig. 3. A variable gain amplifier (VGA) with a tuning range between $12 \mathrm{~dB}$ and $-19.5 \mathrm{~dB}$ is used to adjust the signal level at the input of the 12-bit $500 \mathrm{MHz}$ ADC. Together with the receiver low

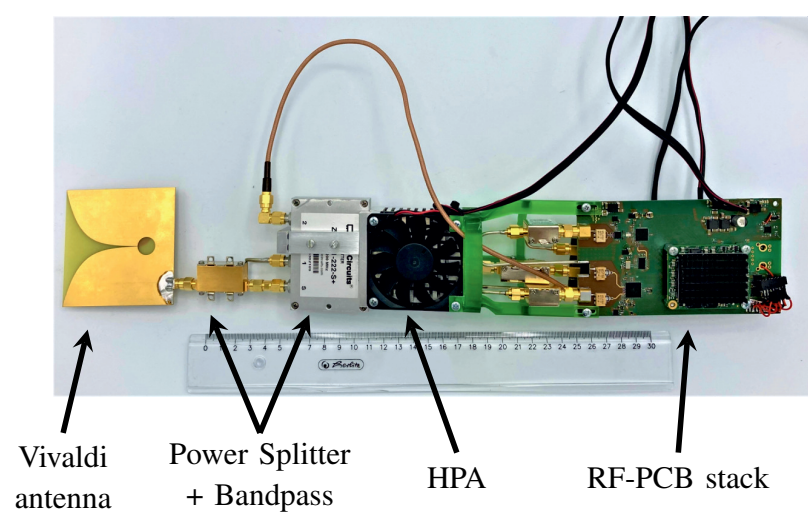

Fig. 4: First prototype of the radar transponder as used in a functional test without power supply and tubular enclosure. Total power consumption is about $20 \mathrm{~W}$ at $24 \mathrm{~V}$.

noise amplifier (LNA) and the active downconversion mixer a maximum gain setting of $46.5 \mathrm{~dB}$ is realized in the $\mathrm{Rx}$ path. Thus, the ADC can use its full dynamic range for input levels at the antenna between $-8.5 \mathrm{dBm}$ and $-40 \mathrm{dBm}$ which is lower than the expected level for an unobstructed F-SAR L-band acquisition at a flight height of $3000 \mathrm{~m}$. The ADC samples are stored in a digital signal processing (DSP) block and output by the 12 -bit $500 \mathrm{MHz}$ DAC after a predefined delay. Both ADC and DAC operate based on the same clock. The pulse is upconverted by the same local oscillator as in the Rx path to ensure phase integrity before amplifying the signal with a high power amplifier (HPA). Switching off the output is realized in a digital manner in the DSP. For achieving the maximum output power and therefore, a maximized effective RCS it is not necessary to fully saturate the ADC as the output path can provide a saturated output power $P_{\text {sat }}=30 \mathrm{dBm}$ and has a total gain of $40 \mathrm{~dB}$ in order to compensate lower input signals. As a result of the remarkable maximum gain of $86.5 \mathrm{~dB}$, a saturated output power can be achieved for input signals as low as $-56.5 \mathrm{dBm}$ which can mitigate signal attenuation caused by the propagation through the ice. Consequently, $G_{\text {sys }}$ is depending on the actual input power at the antenna

$$
G_{\text {sys }}\left(P_{\text {in }}\right)=\left\{\begin{array}{lll}
G_{\text {max }} & \text { for } & P_{\text {in }} \leq P_{\text {sat }}-G_{\text {max }} \\
P_{\text {sat }}-P_{\text {in }} & \text { for } & P_{\text {in }}>P_{\text {sat }}-G_{\text {max }}
\end{array}\right.
$$

and theoretically contributes to an RCS of about $42 \mathrm{dBm}^{2}$ in an unobstructed F-SAR L-band acquisition.

As the radar system and the transponder share no common clock source, the timing for opening the transponder receive window is not known. Hence, it is necessary to synchronize the transponder timing to the radar pulse repetition frequency (PRF). This requires a reliable detection of the incoming radar pulses. It is not sufficient to solely rely on a rising edge in the input signal of the transponderas the risk of false detections is too high. Therefore, pulse compression techniques are integrated into the transponder which enable the usage of the system even in areas with strong distortions.

The transponder continuously scans the incoming signal for a radar pulse using pulse compression while the transmitter 


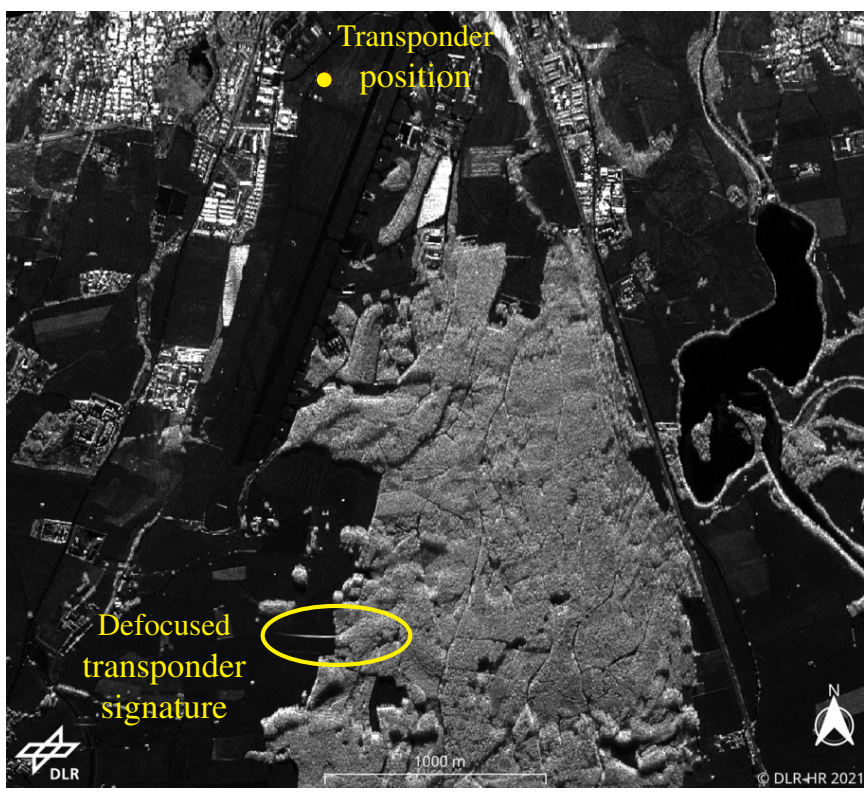

(a)

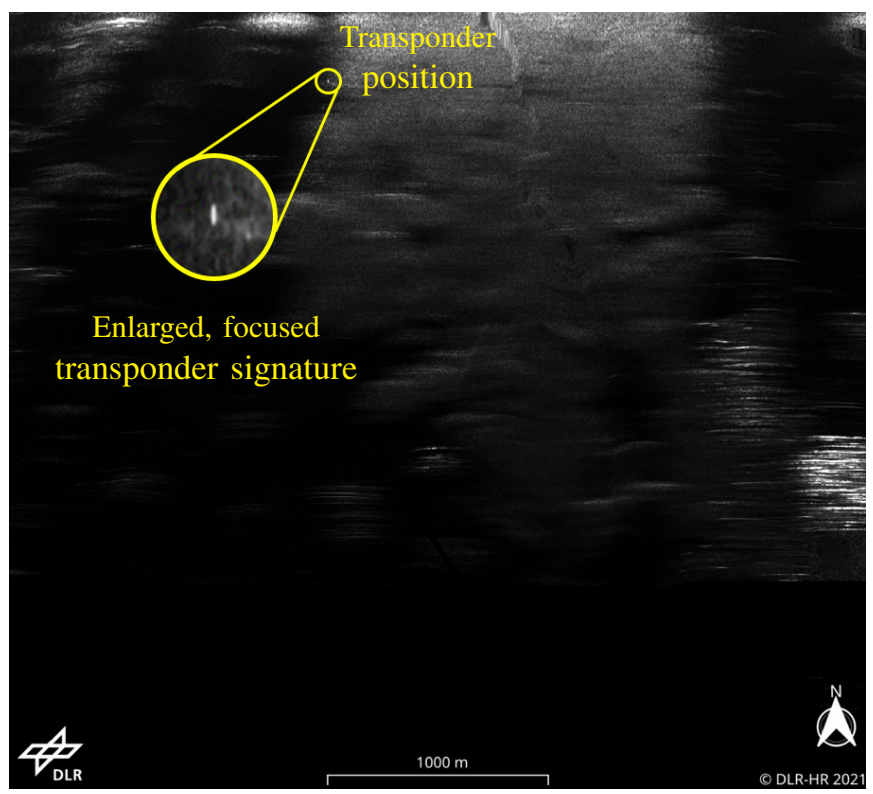

(b)

Fig. 5: Processed data in HH-polarization of a linear flight track illuminating the scene from north.As a worst-case scenario, a delay of $12.06 \mu \mathrm{s}$ was chosen to shift the transponder signature in a complex clutter surrounding. (a) SAR image with neglected transponder delay during SAR focusing. The transponder position is marked with a yellow dot. The defocused transponder signature is highlighted. (b) Same scenery with incorporated transponder delay during SAR focusing. The resulting peak in the defocused clutter from the forest is marked and enlarged with a yellow circle.

is fully muted. Once a pulse is detected, the transponder enters its normal operating mode by periodically switching the transmitter on and off depending on the delay settings and the detected radar PRF. As the clock frequencies of the radar system and the transponder are not perfectly identical it is mandatory to recurrently correct the relative time shift between both. If this effect is neglected, the two clocks drift apart and the receive window timing does not correspond with the incoming radar pulses. The regular timing synchronization is performed every $25 \mathrm{~ms}$. This re-synchronization inherently takes into account the variable signal travel time introduced by the range migration during a SAR acquisition.

\section{Measurement And Results}

In June 2021, a functional test prior to the final utilization in the glacial environment has been carried out at the F-SAR calibration site at the airport in Kaufbeuren, Germany. The configuration of the F-SAR L-band pulsed radar is shown in Table I. The processed SAR data are shown in Fig. 5. It can be seen that, even with a residual defocusing in azimuth direction due to the uncompensated transponder delay, the transponder signature in Fig. 5a is clearly visible even in high clutter environments such as the forest in the right-most part of the parabola. As expected, the signature is shifted in range direction due to the transponder delay. If the same scenery is focused with adjusted processing parameters according to the defined transponder delay, the parabola-like signature focuses to a point target as can be seen in Fig. 5b. A very bright peak is visible at the transponder position, especially compared to the backscatter magnitude of the surrounding scene that appears defocused due to the adjusted processing parameters. Therefore, it can be concluded that the transponder does not degrade the radar pulse in a manner that prevents a functional azimuth and range compression. As can be seen in Fig. 5b, the point target has a worse resolution in range than in azimuth direction corresponding to the ground resolutions of $2.5 \mathrm{~m}$ in range and $0.6 \mathrm{~m}$ in azimuth selected for the SAR processing.

\section{CONCLUSION}

A prototpye of a monostatic digital delay radar transponder with nearly $90 \mathrm{~dB}$ coherent pulse signal amplification for SAR applications was presented in this letter. The small and independently working system achieves excellent performance as it can be clearly detected by an airborne SAR sensor.

Further system characterization tests will be carried out for in-depth analysis of the transponder parameters such as the RCS and the impulse response in range and azimuth. This is relevant for enhanced signal processing. Furthermore, it is planned to upgrade the antenna to a polarization-independent operation allowing for an arbitrary positioning of the transponder in a melting hole. A further test in a glacial environment is planned for March 2022.

\section{ACKNOWLEDGMENT}

This study was partly financially supported by the German Space Agency at the German Aerospace Center (DLR) with funds from the German Federal Ministry for Economic Affairs and Energy (BMWi) under the project EnEx-AsGAr, registration no. 50NA1708. 


\section{REFERENCES}

[1] K. Konstantinidis, C. L. Flores Martinez, B. Dachwald, A. Ohndorf, P. Dykta, P. Bowitz, M. Rudolph, I. Digel, J. Kowalski, K. Voigt, and R. Förstner, "A lander mission to probe subglacial water on Saturn's moon Enceladus for life," Acta Astronautica, vol. 106, pp. 63-89, 2015.

[2] B. Dachwald, M. Feldmann, C. Espe, E. Plescher, K. Konstantinidis, and R. Forstner, "Enceladus Explorer - A maneuverable subsurface probe for autonomous navigation through deep ice," Proceedings of the International Astronautical Congress, IAC, vol. 3, pp. 1756-1766, 01 2012.

[3] D. Heinen, P. Linder, S. Zierke, and C. Wiebusch, "An efficient melting probe for glacial research," Annals of Glaciology, vol. 62, pp. 1-4, 05 2020.

[4] A. Moreira, G. Krieger, M. Younis, I. Hajnsek, K. Papathanassiou, M. Eineder, and F. De Zan, "Tandem-L: A mission proposal for monitoring dynamic earth processes," in 2011 IEEE International Geoscience and Remote Sensing Symposium, 2011, pp. 1385-1388.

[5] M. Arkett, D. Flett, R. De Abreu, P. Clemente-Colon, J. Woods, and B. Melchior, "Evaluating ALOS-PALSAR for ice monitoring - what can L-band do for the North American Ice Service?" in IGARSS 2008 2008 IEEE International Geoscience and Remote Sensing Symposium, vol. 5, 2008, pp. V - 188-V - 191.

[6] S. V. Nghiem and P. Clemete-Colon, "Arctic sea ice mapping with satellite radars," in 2008 IEEE Radar Conference, 2008, pp. 1-3.

[7] A. Reigber, R. Scheiber, M. Jager, P. Prats-Iraola, I. Hajnsek, T. Jagdhuber, K. P. Papathanassiou, M. Nannini, E. Aguilera, S. Baumgartner, R. Horn, A. Nottensteiner, and A. Moreira, "Very-high-resolution airborne synthetic aperture radar imaging: Signal processing and applications," Proceedings of the IEEE, vol. 101, no. 3, pp. 759-783, 2013.

[8] M. Jirousek, B. Doering, D. Rudolf, S. Raab, and M. Schwerdt, "Development of the highly accurate DLR Kalibri Transponder," in EUSAR 2014; 10th European Conference on Synthetic Aperture Radar, 2014, pp. 1-4.

[9] J. Hong, Y. Wang, M. Yi, Y. Zhu, L. Li, and F. Ming, "A revised polarimetric calibration method for GF-3 SAR with active radar transponder," in 2017 Progress in Electromagnetics Research Symposium - Fall (PIERS - FALL), 2017, pp. 1055-1058.

[10] D.-J. Park, S.-I. Ahn, Y.-S. Chun, J.-M. Shin, J.-C. Yoon, and J.-H. Kim, "Development of active transponder for KOMPSAT-5 mission," in 2011 3rd International Asia-Pacific Conference on Synthetic Aperture Radar (APSAR), 2011, pp. 1-4.

[11] A. M. Büchner, K. Weidenhaupt, M. Limbach, S. Raab, and M. Schwerdt, "DLR's innovative reference target development for future SAR missions," in 2020 German Microwave Conference (GeMiC), 2020, pp. 176-179.

[12] R. Horn, A. Nottensteiner, A. Reigber, J. Fischer, and R. Scheiber, "FSAR - DLR's new multifrequency polarimetric airborne SAR," in 2009 IEEE International Geoscience and Remote Sensing Symposium, vol. 2, 2009, pp. II-902-II-905.

[13] M. Porranzl, C. Wagner, H. Jaeger, and A. Stelzer, "Highbandwidth quasi-circulator-based monostatic automotive radar with selfinterference suppression," IEEE Transactions on Microwave Theory and Techniques, vol. 69, no. 1, pp. 198-209, 2021.

[14] D. Rudolf, S. Raab, B. J. Döring, M. Jirousek, J. Reimann, and M. Schwerdt, "Absolute radiometric calibration of the novel DLR "Kalibri" transponder," in 2015 German Microwave Conference, 2015, pp. 323-326. 